\title{
Newborn screening grows up
}

T he effective treatment of inherited disorders afflicting infants is one of the great success stories of modern medicine. For many of these conditions, therapy is available based on our understanding of the culprit mutation and of the resulting biochemical abnormalities. But the timing of diagnosis is crucial: these disorders are all too often not apparent before disability or death has occurred, so they should be screened for in the first few days after birth.

In the US, Massachusetts mandated the first test for newborns, for phenylketonuria, in the early 1960s. Since then, more than 150 million infants have been screened for an increasing number of diseases. The American Academy of Pediatrics estimates that of the 4.1 million infants born each year in the US, 5,000 have conditions that would benefit from screening and treatment, but one-fifth of such cases go undetected. This can largely be attributed to varying requirements among individual states for the number of diseases tested - as of the beginning of this year, some states mandated screening for as few as four disorders, others for more than 30. National guidelines are sorely needed to rectify these disparities.

This March, the American College of Medical Genetics (ACMG) issued guidelines recommending uniform screening among states. The ACMG assembled a panel including public health experts, geneticists, primary care physicians, ethicists, legal specialists and representatives of families to systematically develop criteria and prioritize disorders for newborn screening. These criteria were that a reliable test exists, that the cause and progression of the disease is adequately understood, and that an effective treatment is available. After soliciting expert opinion and reviewing the medical literature, the panel selected 29 conditions meeting these criteria.

The selected conditions include disorders affecting organicacid, fatty-acid and amino-acid metabolism, hemoglobinopathies and several others. In the process of screening for these conditions, results allowing diagnosis of another 25 conditions also become available. As information on these secondary conditions could have clinical implications, the panel recommended that these results should also be made available to doctors and families.

Screening, however, is only the first step in treating these conditions. To minimize potential harm caused by false positive results, it will be crucial to have rapid and effective followup testing. For example, sequencing of the relevant disease gene can provide a conclusive diagnosis for many conditions. Also, some of the disorders included in the screening panel are rare and may not be well understood by primary care physi- cians who provide treatment or by genetic counselors who educate parents. It will therefore be important to disseminate information on these disorders, and the report has taken a step toward that goal by including a fact sheet on each condition.

To be sure, not everyone is satisfied with the panel's recommendations (see page 1020). The conditions selected for testing range from those for which the benefits of screening and treatment are beyond doubt-such as phenylketonuria and congenital hypothyroidism - to those for which the potential benefits and risks are much less clear. In assessing the benefit of screening for some disorders, critics feel that the panel relied too much on expert opinion rather than on hard evidence in the medical literature. Some diseases recommended for screening are extremely rare, with an incidence on the order of 1 in 100,000 births or less; these pose a particular problem, as information about these conditions is limited, including uncertainty about their true incidence and whether treatment is effective.

Addressing these concerns will require a coordinated effort to track screening results and the outcomes of diagnosed babies. For conditions that are less well understood, it will be crucial to track the rate of false positives and whether treatments are actually effective. Testing for rare diseases should provide better numbers for their incidence, and identifying more infants with such conditions might aid research on these disorders.

Some conditions may have been previously underdiagnosed and contribute more than realized to sickness or death in infants. More information on the poorly understood secondary set of disorders should be particularly valuable in contributing to a better understanding of the disease process and the development of treatment. However, collecting these types of data may run afoul of privacy regulations, and it will be important to devise a strategy that allows data collection while addressing legitimate privacy concerns.

Although there may be disagreements among experts about some of the specifics of the ACMG report's recommendations, in the few months since the report was issued, states have taken notice. Most are expanding their newborn screening programs, and 10 already mandate screening for all 29 recommended conditions. It will clearly take significant resources for states to expand screening - to equip clinical laboratories, train laboratory personnel, pay for screening and follow-up testing, and to track results. But standardizing testing across states so that accidents of geography do not have lifeand-death consequences seems like an idea whose time has come. 